\title{
Confirmation of Carnosine and Its Methylated Compounds in the Muscles of Some Animals
}

\author{
Michizo Suyama and Michie Maruyama* \\ (Received March 18, 1969)
}

Carnosine ( $\beta$-alanylhistidine) and its methylated compounds occur widely in the muscles of many species of animals. Anserine ( $\beta$-alanyl-1-methylhistidine**) was first detected in the goose ${ }^{1 /}$ and later in many species $^{2,3)}$. Ophidine $(\beta$-alanyl-2-methylhistidine $^{* *}$ ) has been reported to be present in the cobra and some other snakes ${ }^{4-91}$. Finally, balenine ( $\beta$-alanyl-3-methylhistidine**) was recognized in the meat extract of whales ${ }^{10 \sim 12}$, and proved to be identical with cetasine ${ }^{11,131}$.

Tsunoo et al. ${ }^{14)}$ recently reported the presence of ophidine in the three species of dolphin, Tursiops truncatus, Grampidelphis griseus, and Feresa attenuata, on the basis that the infrared absorption spectra and melting point agreed with those of ophidine from the snakes. The authors ${ }^{15}$ have determined the amounts of carnosine, anserine and balenine, together with the constitutional imidazole compounds, in the extractives of whale meat by the chromatographic technique using an Amberlite IR-120 column $(0.9 \times 50 \mathrm{~cm})$. However, the indication showing the occurrence of ophidine and 2-methylhistidine in the Odontoceti has not been observed so far on the chromatograms.

The present investigation was undertaken to identify the main imidazole dipeptide in the muscles of several kinds of animal. The peptide preparations obtained from the extractives of snake and dolphin by the procedure of NAKAI et al. ${ }^{16)}$ and from those of dolphin by the method of TsuNoo et al. ${ }^{14)}$ showed the same infrared absorption spectrum as of ophidine described by Tsunoo et al. ${ }^{14)}$ It has become clear from nuclear magnetic resonance (NMR) study that the peptide is $\beta$-alanyl-3-methylhistidine and not $\beta$-alanyl-2methylhistidine. The details are given below.

\section{Experimental}

Preparation of carnosine and its methylated compounds from the animal meat. By the procedure of NAKAI et al. ${ }^{18)}$, carnosine and anserine were prepared from the histidine plus arginine fraction of the meat extracts of common dolphin, Delphinus delphis, and from the

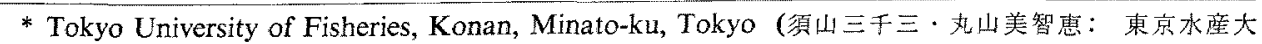
学).

** The trivial names "1-methylhistidine, 2-methylhistidine, and 3-methylhistidine" are inconsistent with the accepted numbering method for imidazole derivatives, the systematic names being $\alpha$-amino- $\beta$-(1methyl-5-imidazoyl)propionic acid, $\alpha$-amino- $\beta$-(2-methyl-4(or 5)-imidazoyl)propionic acid, and $\alpha$-amino$\beta$-(1-methyl-4-imidazoyl)propionic acid, respectively. We have, however, used the trivial names for brevity. 
lysine fraction of those of bigeye tuna, Parathunnus mebachi, respectively. Two specimens of balenine were obtained from the sei whale, Balaenoptera borealis, and the common dolphin, and two specimens of ophidine from the snakes, Natrix tigrina and Elaphe quadrivirgata, respectively. The snake, $N$. tigrina, has been reported to contain ophidine by TAKEDA $^{9}$. Beside these, the meat of common dolphin was treated by the method of Tsunoo et al. ${ }^{14)}$ in order to obtain ophidine.

All these specimens were prepared as free base, and recrystallized from ethanol in clumps of fine needles. They were hygroscopic to some extent, and stored in a dehydrated state.

Measurement of infrared absorption spectra. An infrared spectrometer (Nihon Bunko Kogyo, Model IR-S) was used throughout this experiment.

Mesurement of NMR spectra. The proton resonance spectra were measured by a Varian HA-100 NMR spectrometer operated at $100 \mathrm{Mcps}$, using deuterium oxide solution and sodium 2,2-dimethyl-2-silapentane sulfate (DSS) as solvent and internal reference, respectively.

\section{Results}

Properties of the specimens. Carnosine gave a red color with Pauly's reagent, but the other specimens gave no color. Elemental analyses showed the approximate formulae, $\mathrm{C}_{9} \mathrm{H}_{14} \mathrm{~N}_{4} \mathrm{O}_{3}$ for carnosine, and $\mathrm{C}_{10} \mathrm{H}_{10} \mathrm{~N}_{4} \mathrm{O}_{3} \cdot{ }_{1} /{ }_{2} \mathrm{H}_{2} \mathrm{O}$ for the others. The melting points of the specimens determined by the use of prismscopic apparatus (Mitamura Riken Kogyo Co. Ltd.) were $229-231^{\circ} \mathrm{C}$ for carnosine, $224^{\circ} \mathrm{C}$ for anserine, and $222-224^{\circ} \mathrm{C}$ for the others.

Grouping of specimens by infrared absorption spectra. The infrared absorption spectra observed are shown in Fig. 1, and three types of spectrum are apparent, respectively represented by carnosine, anserine and the others. Among the five samples from No. 3 to 7 , including balenine and ophidine, no marked difference is recognized. It may be noteworthy that TsuNOo et al. ${ }^{14)}$ obtained a quite identical infrared absorption spectrum on their ophidine preparations with that of balenine reported by NAKAI and TSUJIGADO ${ }^{11}$ but they did not notice the identity.

NMR signals of carnosine. The NMR spectrum of carnosine together with peak assignments is shown in Fig. 2.

The spectrum is analyzed from chemical shift and line shape as follows: The resonance lines at 7.50-6.70 $\tau$ are due to the four protons $\left(\mathrm{H}_{\mathrm{a}}, \mathrm{H}_{\mathrm{a}^{\prime}}, \mathrm{H}_{\mathrm{b}}\right.$, and $\left.\mathrm{H}_{\mathrm{b}^{\prime}}\right)$ of two $\mathrm{CH}_{2}$ groups of the $\beta$-alanyl residue and to the two protons $\left(\mathrm{H}_{\mathrm{d}}\right.$ and $\left.\mathrm{H}_{\mathrm{e}}\right)$ of $\beta-\mathrm{CH}_{2}$ of the histidine moiety. Owing to the asymmetric $\alpha$-carbon of histidine, $H_{d}$ should not be equivalent to $H_{e}$, and the pattern is considered as the $\mathrm{AB}$ portion of an $\mathrm{ABX}$ system. The quartet at $5.55 \tau$ is due to $\mathrm{H}_{\mathrm{c}}$ of the $\alpha$-carbon of histidine. As illustrated in spin-decoupling ( $445 \mathrm{cps}$, denoted by (1) in Fig. 2), $H_{c}$ is coupled to $H_{d}$ and $H_{e}$, and gives a quartet assignable to the $X$ portion 


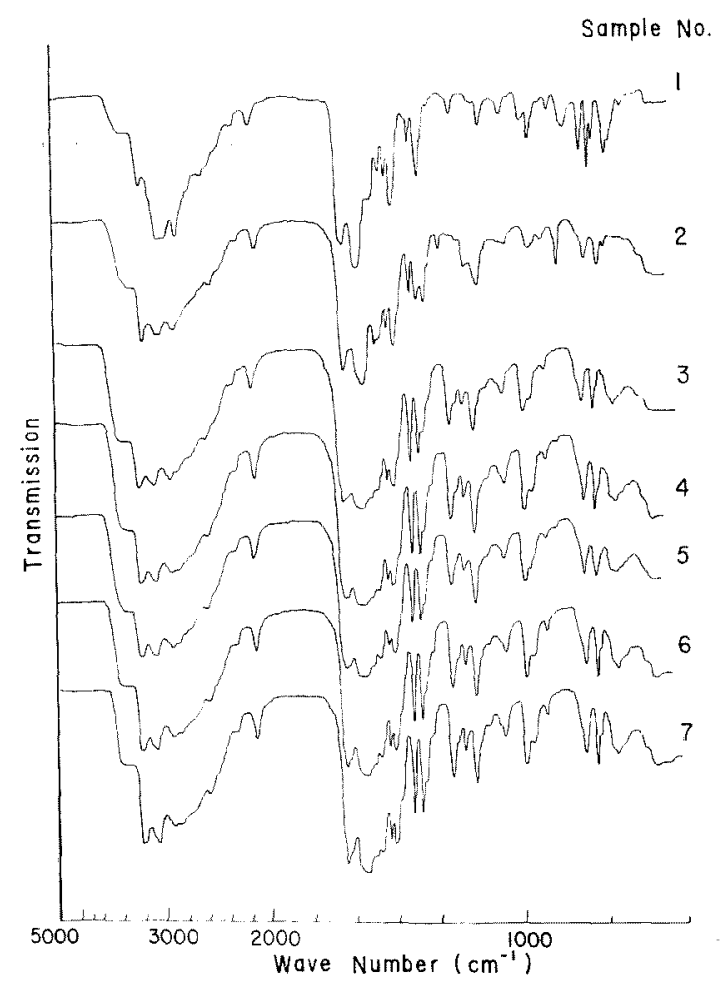

Fig. 1. Infrared absorption spectra of carnosine and its methylated compounds in $\mathrm{KBr}$.

No. 1: Carnosine from common dolphin. No. 2: Anserine from bigeye tuna. No. 3: Balenine from sei whale. No. 4: Balenine from common dolphin. No. 5: Ophidine from common dolphin. No. 6: Ophidine from Natrix tigrina. No. 7: Ophidine from Elaphe quadrivirgata.

Specimens No. 1-4, 6 and 7 were prepared by the method of NAKAI $e f$ al. and specimen No. 5 by the method of Tsunoo et al.

of an ABX system. The strong peak at $5.20 \tau$ is attributed to the presence of HDO; the exchangeable protons of $\mathrm{NH}, \mathrm{NH}_{2}$, and $\mathrm{COOH}$ are displaced by deuterium and their resonances are included in the peak.

The two protons of the imidazole moiety, $\mathrm{H}_{\mathrm{f}}$ and $\mathrm{H}_{\mathrm{g}}$, show absorptions at $3.07 \tau$ and $2.30 \tau$, and the respective positions are decided by spin-decoupling as follows: The peaks at $2.30 \tau$ and $3.07 \tau$ are converted to sharp peaks by irradiations of $693 \mathrm{cps}$ (denoted by (2) in Fig. 2) and of $770 \mathrm{cps}$ (denoted by (3) in Fig. 2), respectively; the two peaks are mutually coupled. Irradiation of $693 \mathrm{cps}$ (2) shows a distinct influence on the resonance lines at

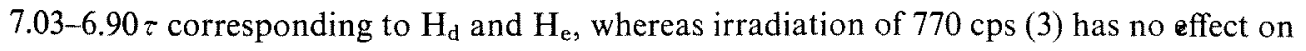
the signals of $\mathrm{H}_{\mathrm{d}}$ and $\mathrm{H}_{\mathrm{e}}$.

From the formula of carnosine, it may be assumed that the coupling constants $J_{\mathrm{d}, \mathrm{f}}$ 


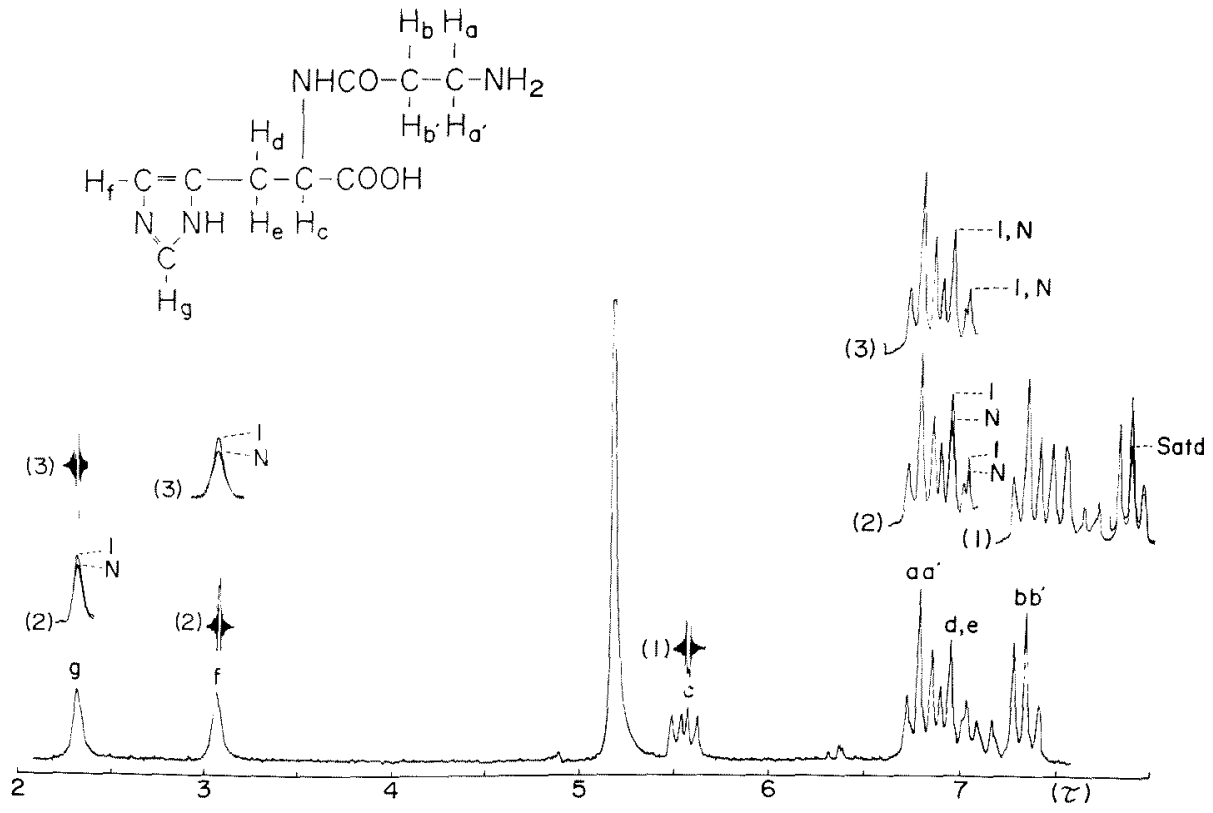

Fig. 2. NMR spectra of carnosine prepared from the muscle of common dolphin. Irradiations of $H_{c}, H_{f}$ and $H_{g}$ are denoted by (1), (2) and (3), and, the heights of resonances before and after irradiations are by N and I, respectively. Spectrometer, Varian HA-100; internal standard, DSS; solvent, $\mathrm{D}_{2} \mathrm{O}$; sweep time, $500 \mathrm{sec}$; sweep width, 500 cps.

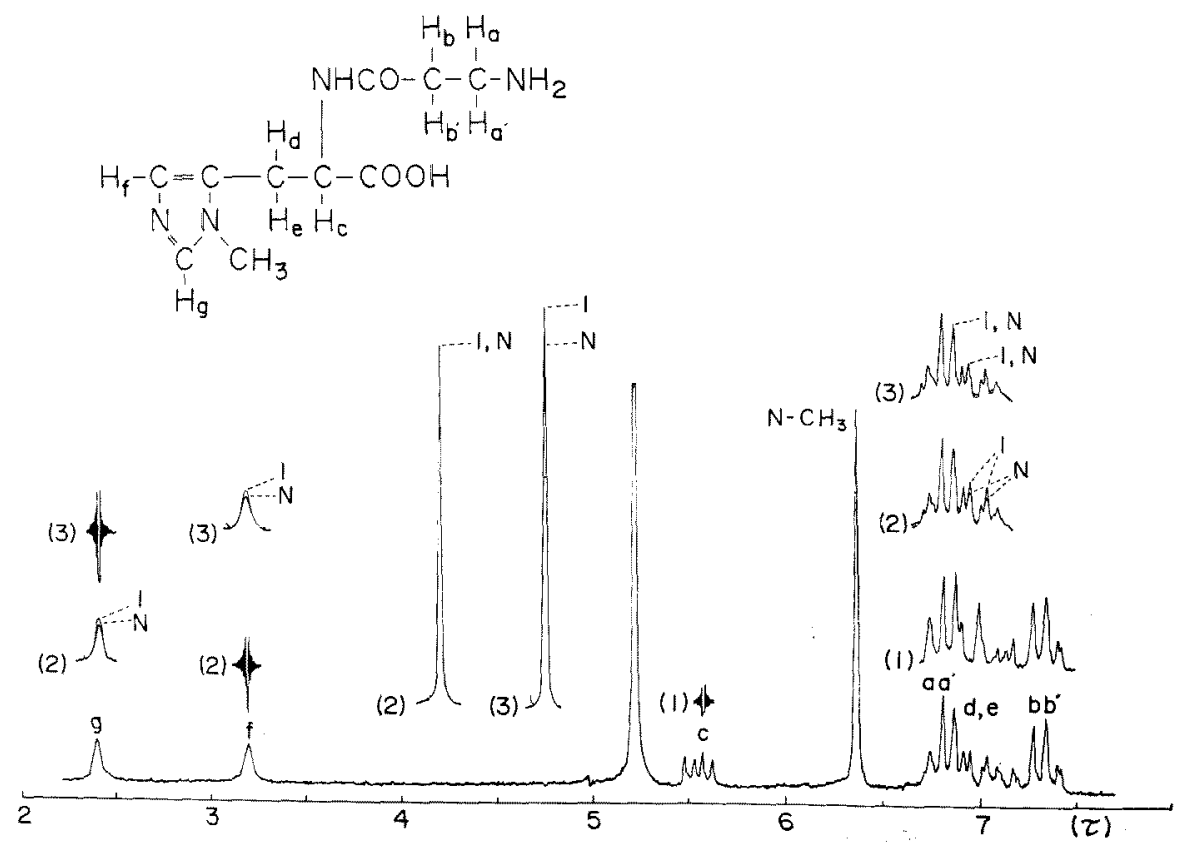

Fig. 3. NMR spectra of anserine prepared from the muscle of bigeye tuna. 


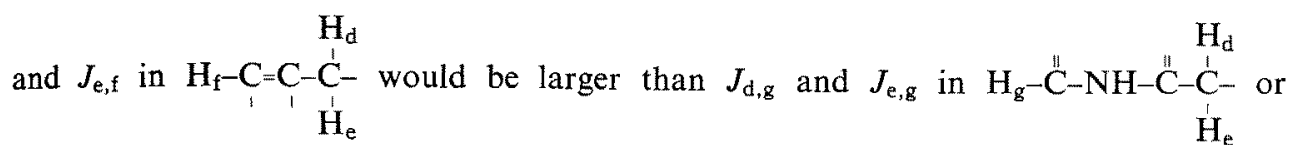
$\mathrm{H}_{\mathrm{d}}$

$\mathrm{H}_{\mathrm{g}}-\stackrel{\dot{C}}{\mathrm{C}}=\mathrm{N}-\stackrel{\text { CH}}{\mathrm{C}}-\mathrm{C}-$. The signals of $\mathrm{H}_{\mathrm{d}}$ and $\mathrm{H}_{\mathrm{e}}$ are thus affected by the irradiation of 693 $\mathrm{H}_{\mathrm{e}}$

cps $\left(\mathrm{H}_{f}\right)$ but little affected by that of $770 \mathrm{cps}\left(\mathrm{H}_{\mathrm{g}}\right)$. These findings indicate that the signal at $3.70 \tau$ is assigned to $H_{f}$ and that at $2.30 \tau$ to $H_{g}$.

NMR signals of anserine. The NMR spectrum of anserine is shown in Fig. 3. The signals at 7.50-6.70 $\tau, 5.55 \tau$ (quartet), and $5.20 \tau$ seem to be in good accordance with the above elucidation on carnosine. The two signals at $3.19 \tau$ and $2.40 \tau$ are assigned to $\mathrm{H}_{\mathrm{f}}$ and $\mathrm{H}_{\mathrm{g}}$ of imidazole by the technique of spin-decoupling, respectively. A sharp peak at $6.36 \tau$ is assigned to the $\mathrm{N}$-methyl group from the evaluation of chemical shift.

The position of the methyl group of imidazole is decided as follows: Irradiation of $\mathrm{H}_{\mathrm{g}}$ (760 cps, denoted by (3) in Fig. 3) heightens the N-methyl peak, but shows almost no

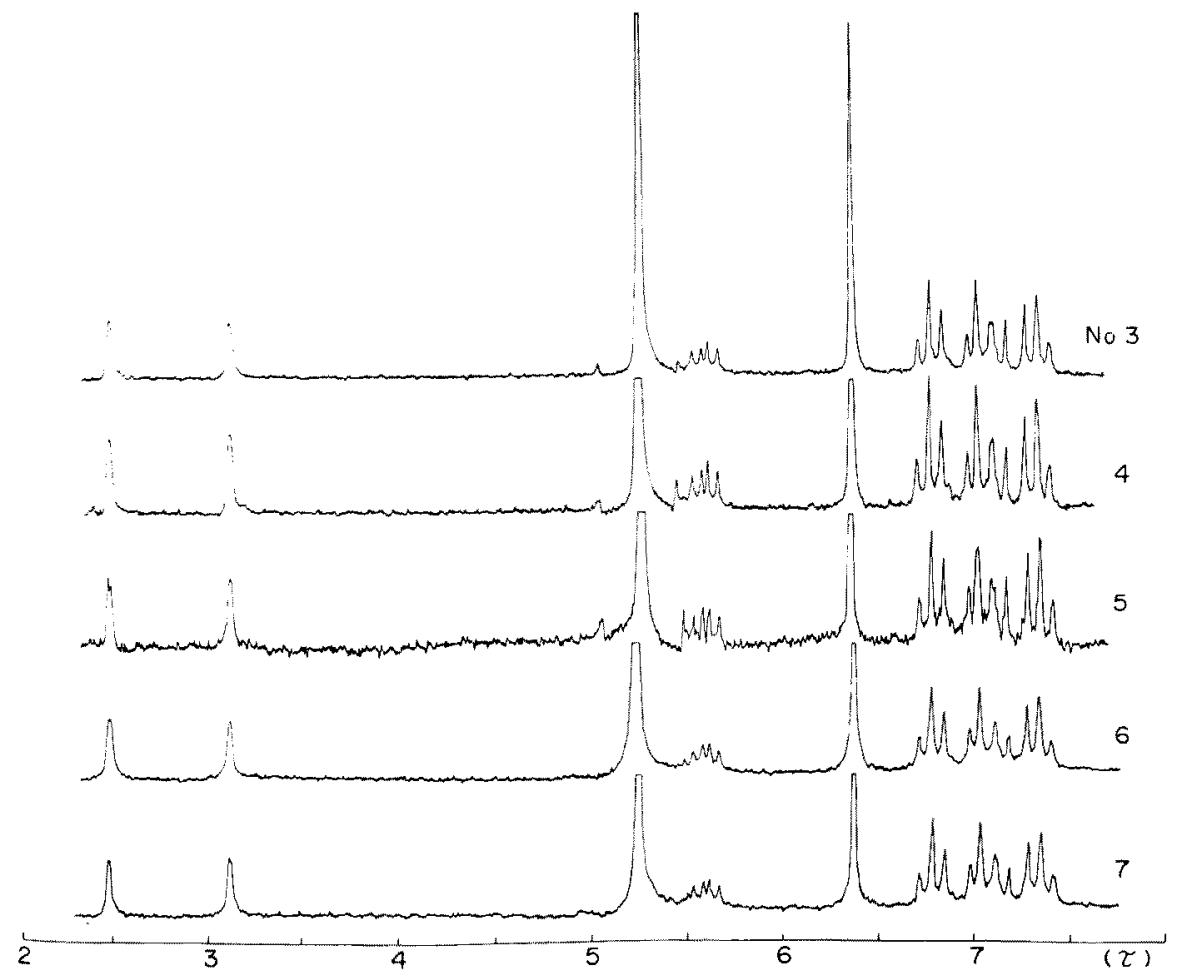

Fig. 4. NMR spectra of balenine and ophidine.

No. 3: Balenine from sei whale. No. 4: Balenine from common dolphin. No. 5: Ophidine from common dolphin. No. 6: Ophidine from Natrix tigrina. No. 7: Ophidine from Elaphe quadrivirgata. 


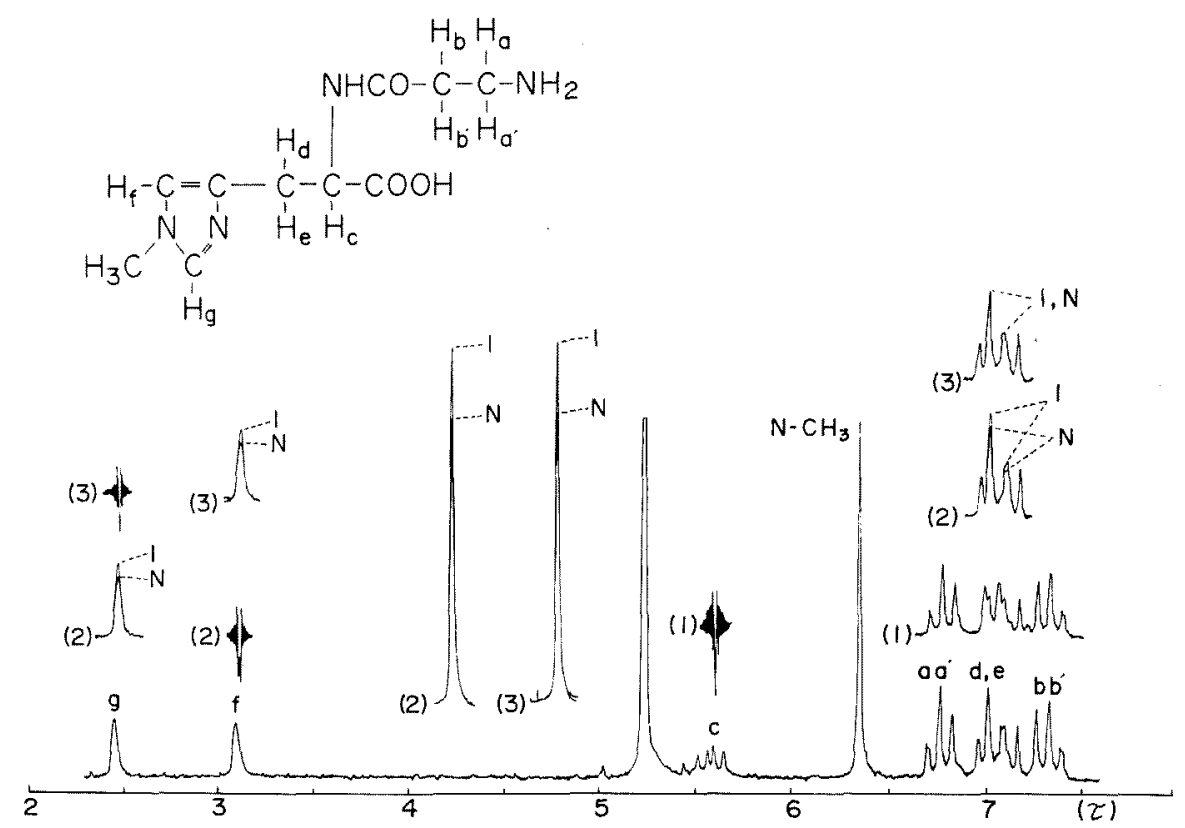

Fig. 5. NMR spectra of balenine prepared from the muscle of sei whale.

effect on the peaks of $H_{d}$ and $H_{e}$. Irradiation of $H_{f}(681 \mathrm{cps}$, denoted by (2) in Fig. 3) has no effect on the peak of $\mathrm{N}$-methyl, but heightens the peaks of $\mathrm{H}_{d}$ and $\mathrm{H}_{e}$. These findings mean that $\mathrm{H}_{\mathrm{g}}$ is far from $\mathrm{H}_{\mathrm{d}}$ and $\mathrm{H}_{\mathrm{e}}$, but not far from $\mathrm{N}$-methyl; and that $\mathrm{H}_{\mathrm{f}}$ is not far from $\mathrm{H}_{\mathrm{d}}$ and $\mathrm{H}_{\mathrm{e}}$, but far from $\mathrm{N}$-methyl. Therefore, the structure of anserine is unequivocally expressed as 1-methyl compound.

NMR signals of balenine and ophidine. The NMR spectra of balenine and ophidine are summarized in Fig. 4, in which five specimens show identical NMR spectra. The data led to a problem as to which compound shows such a spectrum, the 3-methyl compound or 2-methyl compound.

To solve a question, the signals were analyzed by spin-decoupling as follows (Fig. 5): The resonance lines at 7.50-6.70 $\tau, 5.58 \tau$ (quartet), and $5.20 \tau$ are interpreted in the same way as described above. The signals at $6.35 \tau, 3.10 \tau$, and $2.46 \tau$ are assigned to be $\mathrm{N}$ methyl, $\mathrm{H}_{\mathrm{f}}$, and $\mathrm{H}_{\mathrm{g}}$, respectively. Irradiation of $\mathrm{H}_{\mathrm{f}}((2)$ in Fig. 5) makes the resonance line of $\mathrm{N}$-methyl higher, and also irradition of $\mathrm{H}_{\mathrm{g}}((3)$ in Fig. 5) makes it higher in the same degree. It may therefore be concluded that the $\mathrm{N}$-methyl is located between $\mathrm{H}_{\mathrm{f}}$ and $\mathrm{H}_{\mathrm{g}}$, and this allows one to identify the moiety $\mathrm{H}_{\mathrm{f}}-\stackrel{\mathrm{C}}{\|}-\mathrm{N}-\mathrm{C}-\mathrm{H}_{\mathrm{g}}$, a 3-methyl compound (balenine).

$\mathrm{CH}_{3}$

\section{Discussion}

Ophidine was reported to be present in the muscle of cobra by IMAMURA ${ }^{4}$, and to be 
$\beta$-alanyl-2-methylhistidine by $\mathrm{KENDO}^{5}$, since it exhibited the positive Pauly's reaction beside some other features. Since Pauly's reaction is, in the case of imidazole compound, known to develop only in the compounds in which the NH group remains in the free state ${ }^{17}$, the methyl group had thus been assumed to be located at 2-position of the imidazole ring. ONO ${ }^{18)}$ and ONO and HIROHATA ${ }^{19)}$ have recognized that chemically synthesized 2-methylhistidine was strongly positive in the reaction.

However, there have been conflicting data on this subject. $\mathrm{KENDO}^{57}$ and TAKEDA ${ }^{97}$ have reported that both specimens of ophidine prepared from the cobra and Natrix tigrina gave the weak but positive Pauly's reaction, and Tsunoo et al. ${ }^{7,8)}$ have shown that the specimens obtained from the snake, Trimeresurus flavoviridis flavoviridis, and from the sea snakes, Laticauda semifasciata and L. laticaudata, were negative in the reaction.

Putting these mentions together, the authors have some doubts about the purity of the specimens, and presume that the positive reaction to Pauly's reagent may be due to the presence of impurities such as carnosine (e.g.: The muscle of Natrix tigrina contains 209 $\mathrm{mg} \%$ carnosine in addition to $111 \mathrm{mg} \%$ balenine $^{20)}$.). In fact, the purified specimen of balenine gave a negative reaction in the present study.

The misconception of ophidine is, also, attributable to ONO's report in which the same $\mathrm{Rf}$ value was given to the synthesized $\beta$-alanyl-2-methylhistidine and the preparations from the snake. To state, the identification of carnosine and its methylated compounds by the technique of paper chromatography seems to be difficult, because these compounds give very close $\mathrm{Rf}$ values and are not distinguishable by the $\mathrm{Rf}$ values and the color developed with ninhydrin reagent. The same difficulty was also recognized in thin layer chromatography ${ }^{20)}$.

Summing it up, the authors are positive in denying the existence of $\beta$-alanyl-2-methylhistidine in the muscles of snakes and common dolphin. It is apparent that the compound so far designated as ophidine is nothing but identical with balenine ( $\beta$-alanyl-3methylhistidine). If the previous assumption on $\beta$-alanyl-2-methylhistidine is true, the NMR spectrum due to the 2-methylimidazole moiety should be evidently different from 1- or 3-methyl compound in the number of $\mathrm{CH}$ protons; 2-methylimidazole should have one $\mathrm{CH}$ proton as opposed to 1 - or 3-methylimidazole having two $\mathrm{CH}$ protons. Such a spectrum was not obtained on the specimens analyzed in the present investigation.

WolfF et al. ${ }^{21)}$ recently reported that the structure of ophidine, obtained from the whale meat (species is not clarified), reveals the presence of 3-methyhistidine moiety on the basis of the comparison of NMR spectrum of sample with those of 1-, 2-, and 3-methylhistidine as well as anserine.

The authors wish to express their sincere thanks to Central Research Laboratories, Ajinomoto Co., Inc. for technical assistance on NMR spectral study. 


\section{References}

1) D. Ackermann, O. Timpe, and K. Poller: Z.physiol. Chem., 183, 1-10 (1929).

2) W. A. Wol.F and D. W. Wilson: J. Biol. Chem., 95, 495-504 (1932); 109, 565-571 (1935),

3) J. A. ZAPP and D. W. Wilson: ibid., 126, 9-18, 19-27 (1938).

4) H. Imamura: J. Biochem., 30, 479490 (1939).

5) K. Kendo: ibid., 36, 265-276 (1944).

6) T. TOMTTA: Z. physiol. Chem., 304, 72-76 (1956).

7) S. Tsunoo, K. Horisaka, Y. Miyashita, J. Takeda, and N. Takatama: Jap. J. Pharmacol., 14, 401-411 (1964).

8) S. Tsunoo, K. Horisaka, K. Motonisht, and J. TAKedA: J. Biochem., 56, 604-606 (1964).

9) J. TAKEDA: J. Biochem., 57, 1-6 (1965).

10) F. Pocchiari, L. Tentori, and G. Vrvaldi: Sci. Repts. Ist. Super. Sanità, 2, 188-194 (1962).

11) T. NakaI and N. Tsujigado: J. Biochem., 57, 812-814 (1965).

12) P. O. Dennis and P. A. Lorkin: J. Chem. Soc., 1965 (Sept.), 4968-4972.

13) E. Müller: Z. physiol. Chem., 287, 40-42 (1951); 317, 190-192 (1959).

14) S. Tsunoo, K. Horisaka, H. Tanabe, M. Murata, S. Takahashi, A. Musashi, and J. Tanabe: Jap. J. Pharmacol., 16, 98-109 (1966).

15) M. Suyama, T. Suzuki, and J. Nonaka: This Bull., 33, 141-146 (1967).

16) T. Nakai, N. Tsujigado, and S. AkiYa: J. Biochem., 54, $541-549$ (1963); 56, $482-483$ (1964).

17) K. Hofmann: Imidazole and Its Derivatives, Part 1, 136, Interscience Publishers, Inc., New York (1953).

18) T. Ono: J. Jap. Biochem. Soc. (Seikagaku), 27, 278-283 (1955).

19) T. ONo and R. Hirohata: Z. physiol. Chem, 304, 77-81 (1956).

20) M. Suyama: Unpublished data.

21) J. Wolff, K. Horisaka, and H. M. Fales: Biochem., 7, 2455-2457 (1968). 